\title{
All polarization-maintaining fiber laser architecture for robust femtosecond pulse generation
}

\author{
Wolfgang Hänsel ${ }^{1}$ (1) · Heinar Hoogland ${ }^{1,2} \cdot$ Michele Giunta $^{1,3} \cdot$ Sebastian Schmid $^{1,4}$ • \\ Tilo Steinmetz ${ }^{1} \cdot$ Ralf Doubek $^{1} \cdot$ Peter Mayer $^{1} \cdot$ Sven Dobner $^{1} \cdot$ Carsten Cleff $^{1}$. \\ Marc Fischer $^{1} \cdot$ Ronald Holzwarth ${ }^{1,3}$
}

Received: 7 October 2016 / Accepted: 17 November 2016 / Published online: 11 January 2017

(c) The Author(s) 2016. This article is published with open access at Springerlink.com

\begin{abstract}
We report on a novel architecture for robust mode-locked femtosecond fiber lasers using a nonlinear optical loop mirror with all polarization-maintaining fibers. Due to a nonreciprocal phase shift, the loop mirror can be operated in a compact and efficient reflection mode, offering the possibility to reach high repetition rates and easy implementation of tuning elements. In particular, longitudinal mode spacing and carrier-envelope offset frequency may be controlled in order to operate the laser as an optical frequency comb. We demonstrate femtosecond pulse generation at three different wavelengths (1030, 1565, and $2050 \mathrm{~nm}$ ) using Ytterbium, Erbium, and co-doped Thulium-Holmium as gain media, respectively. Robust operation is achieved for a wide range of parameters, including repetition rates from 10 to $250 \mathrm{MHz}$.
\end{abstract}

This article is part of the topical collection "Enlightening the World with the Laser" - Honoring T. W. Hänsch guest edited by Tilman Esslinger, Nathalie Picqué, and Thomas Udem.

Electronic supplementary material The online version of this article (doi:10.1007/s00340-016-6598-2) contains supplementary material, which is available to authorized users.

Wolfgang Hänsel

w.haensel@menlosystems.com

1 Menlo Systems GmbH, Am Klopferspitz 19a, 82152 Martinsried, Germany

2 Department of Physics, University of Erlangen-Nuremberg, Staudtstrasse 1, 91058 Erlangen, Germany

3 Max-Planck-Institute of Quantum Optics, Hans-Kopfermann-Strasse 1, 85748 Garching, Germany

4 Present Address: TSED2, Airbus Defence and Space GmbH, Robert Koch Str. 1, 82024 Taufkirchen, Germany

\section{Introduction}

Over the last decades, mode-locked laser sources emitting ultrashort pulses have enabled an ever growing number of applications, covering diverse fields such as precision spectroscopy, synchronization and timing, two-photon spectroscopy, generation of $\mathrm{THz}$ radiation, study of attosecond dynamics, pure microwave generation, and material processing. In particular, as a source for a frequency comb [1], mode-locked lasers have initiated a new era of technology with diverse applications. While early applications of femtosecond pulses were limited to well-controlled laboratory environments, a huge number of applications require pulse sources which work under less stable, even harsh conditions as might be present in industrial manufacturing halls, vehicles, air planes, and even satellites. Fiber lasers naturally shield the beam path from the environment to a large extent and hence present a promising technology for such applications. However, polarization-maintaining (PM) fiber has to be used to sufficiently decouple the laser from perturbations such as vibration or variation of temperature, humidity, and air pressure.

Two main strategies are typically used to achieve modelocking in fiber lasers: intrinsic saturable absorbers such as semiconductor saturable absorber mirrors (SESAM), carbon nanotubes or graphene on the one hand, and additive pulse mode-locking (APM) [2] on the other hand. While intrinsic absorbers are easily combined with PM fiber, a low damage threshold and potential degradation over time need to be carefully considered in the laser design [3]. Even more, recent studies of an optical frequency comb based on a SESAM mode-locked fiber laser suggest that intrinsic effects of the SESAM such as the slow relaxation cause a noise floor dominating all other noise contributions like pump or technical noise $[4,5]$. APM schemes, on the 

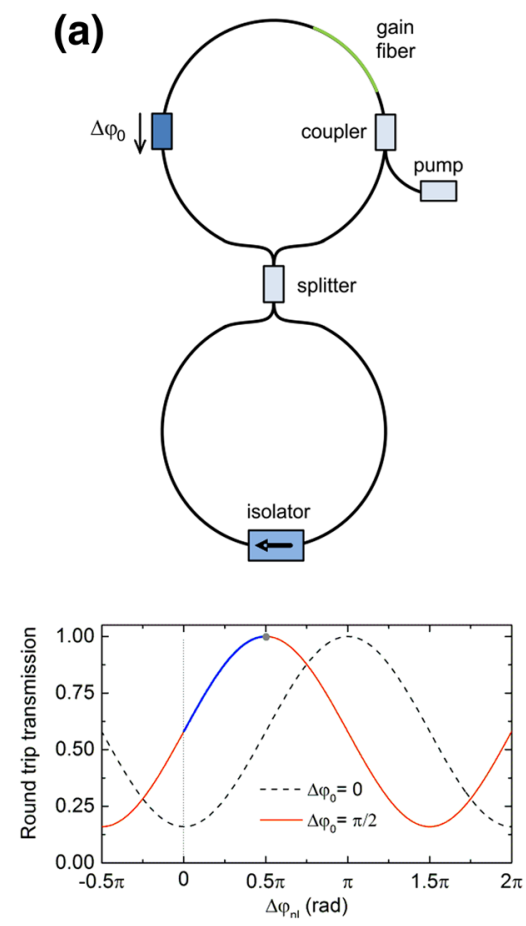
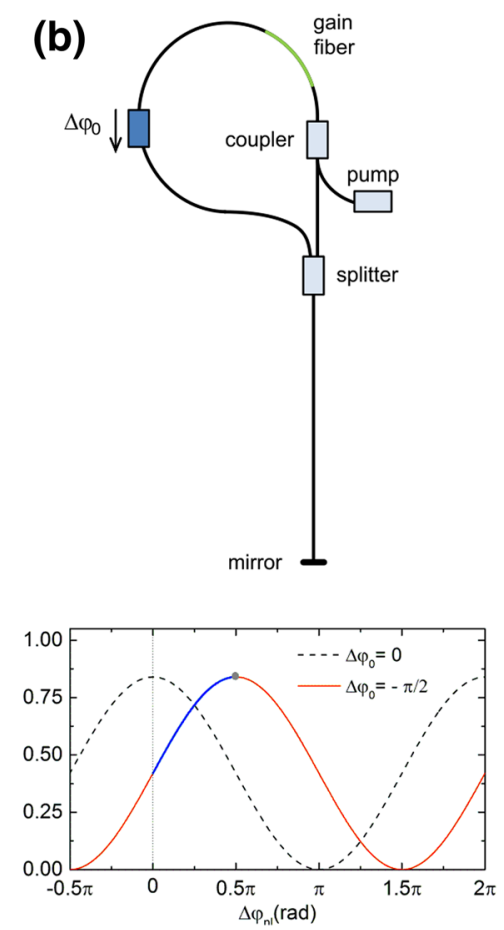

(c)
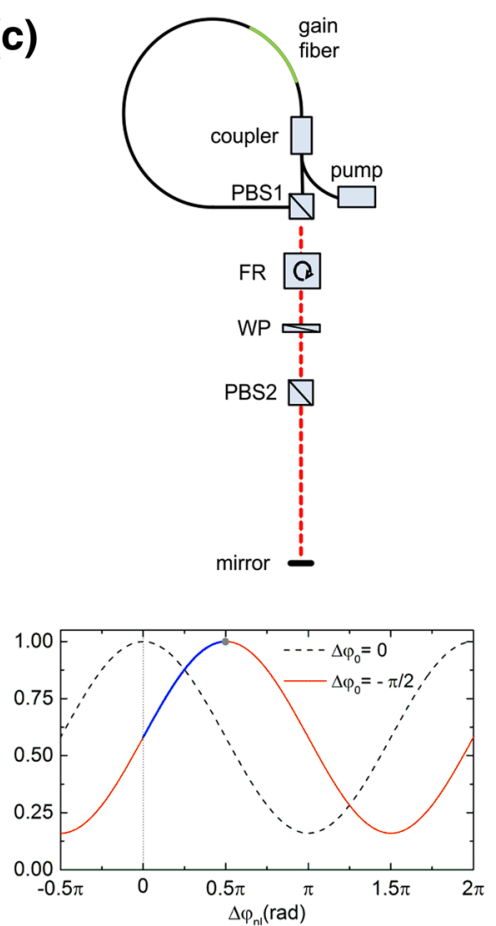

Fig. 1 Schematic of three different laser configurations for additive pulse mode-locking with a nonreciprocally biased NALM. a NALM configuration in transmission, $\mathbf{b}$ reflective NALM configuration with reciprocal beam splitter, c NALM configuration with nonreciprocal beam splitter. For each configuration (top row), the theoretical roundtrip transmission is plotted (bottom row) without (black, dashed) and with (red/blue, straight) nonreciprocal phase bias, assuming an exemplary splitting ratio of 70:30. For the transmission graphs, an exemplary phase bias $\Delta \varphi_{0}=(\pi / 2,-\pi / 2,-\pi / 2)$ in graphs $\mathbf{a}-\mathbf{c}$ has been used, and the corresponding location of mode-locked operation has been marked by a filled circle. The direction of the nonreciprocal phase bias has been defined to be in the direction along which the nonlinear phase shift occurs. The blue part of the round-trip transmission marks the evolution of the laser from continuous-wave (lowintensity) to pulsed (maximum peak power) operation other hand, have been used in fiber lasers since the early 1990s and show the advantage of fast response and therefore potentially lower intrinsic noise. The most prominent examples of APM in fiber lasers are nonlinear polarization rotation (NPR) [6, 7], and a Sagnac loop with intensitydependent transmission [8], often termed nonlinear optical loop mirror (NOLM). To create a mode-locked laser from a NOLM, the loop is naturally operated in transmission. In such a geometry, the connecting fibers form the shape of an " 8 " (see Fig. 1a), and such lasers are also known as figure-eight lasers. The intensity dependence of the NOLM is caused by a differential phase shift between the two counter-propagating pulses (nonlinear nonreciprocal phase shift) which itself is induced by an asymmetry in the loop. While in the original demonstration the asymmetry is induced by the splitting ratio at the NOLM entrance, a popular enhancement of this scheme consists of a loop with an asymmetrically placed amplifying fiber, which is termed nonlinear amplifying loop mirror (NALM) [9].

Due to its nature, NPR cannot be realized in PM fibers. Instead, efforts have recently been taken to implement NALM lasers using PM fiber. While NALM loops with standard (non-PM) fiber offer the possibility to introduce an intrinsic nonreciprocity into the loop using polarization controllers [10], NALM loops with PM fibers are strictly reciprocal for low intensity unless an additional nonreciprocal phase shift element is introduced. Without such nonreciprocal phase bias, the transmission for low intensity is close to zero and does not offer any intensity dependence. This strongly impedes self-starting and has led to efforts to actively start the mode-locking $[11,12]$. Recently, it has been shown by several groups that self-starting ability can be achieved by introducing a nonreciprocal phase bias in the NOLM loop [4, 13, 14] using a proper arrangement of Faraday rotators and waveplates. Even more, the phase bias facilitates operation of the PM-NOLM loop in transmission as well as in reflection. Lasers mode-locked with a reflective NOLM or NALM loop are offered by Menlo Systems $\mathrm{GmbH}$ under the trademark figure $9^{\mathrm{TM}}$.

Unlike a Sagnac loop operated in transmission, a reflective Sagnac loop exhibits a reduced maximum transmission if an asymmetric splitter is used at the junction. Unfortunately, for increasing repetition rates, the asymmetry introduced into a NALM by the amplifying fiber becomes ever 
smaller while the loss from an asymmetric splitting, which might be raised for compensation, becomes increasingly severe. Here we present a novel configuration of a modelocked fiber laser with a reflective all-PM Sagnac loop that combines the low losses of a Sagnac loop in transmission with the advantageous form factor of a reflective configuration. This has led to the demonstration of repetition rates as high as $250 \mathrm{MHz}$, which is about three times higher than results previously reported for all-PM fiber lasers using NALM mode-locking.

\section{NOLM lasers with nonreciprocal phase bias}

A NOLM loop can be integrated into a mode-locked laser in a variety of configurations. Figure 1 compares properties of three different layouts. In the context of this paper, these configurations are considered for single polarization propagation as it is achieved using PM fiber. The upper row shows the laser configuration while the lower row shows the interferometric contribution of the NOLM to the round-trip transmission of a light pulse as a function of the nonlinear differential phase shift $\Delta \varphi_{\mathrm{NL}}$. For the plots, we have assumed an exemplary splitting ratio of 70:30 at the entrance of the NOLM loop. Each of the plots shows the round-trip transmission without a nonreciprocal phase bias (dashed) and with an exemplary nonreciprocal phase bias appropriate for mode-locking operation (straight line). The round-trip transmission of the laser is calculated using the fundamental properties of standard reciprocal beam splitters and, in the third case, using Jones matrices of polarization optics.

The first column (Fig. 1a) shows the popular figure-eight configuration, where the NOLM is operated in transmission, while the other two columns (Fig. 1b, c) represent different reflective configurations. The main difference between the first configuration and the two reflective configurations is that, without any nonreciprocal phase bias (dashed line in the transmission plot), the figure-eight variant exhibits an increasing transmission as a function of the nonlinear phase difference, hence intensity, while the reflective variants both show decreasing transmission. This is why figure-eight lasers are naturally used for passive mode-locking. However, in the presence of an appropriate nonreciprocal phase bias $\Delta \varphi_{0}$, herein chosen as $\Delta \varphi_{0}= \pm \pi / 2$, all configurations can be tuned to increasing intensity (solid line), thus supporting passive mode-locking of the laser. Even more, the phase bias permits to choose a low-intensity point with a nonvanishing slope, which enhances the self-starting of the mode-locking.

It is interesting to note that for all three configurations the contrast of the intensity modulation computes to $c=4(R-0.5)^{2}$, where $R$ represents the reflectance of the NOLM splitter. When using a standard beam splitter for the NOLM (first two configurations), the modulation ranges of the figure-eight and the reflective configurations are offset by $1-c$ with respect to one another, reaching a maximum round-trip transmission of unity for the figureeight case (Fig. 1a) and of $c$ in the reflective configuration with a standard beam splitter (Fig. 1b). In the third column (Fig. 1c), we show our novel reflective configuration which replicates the maximum transmission of 1 inherent to the figure-eight configuration. This feature is achieved by a nonreciprocal beam splitter consisting of a $45^{\circ}$-Faraday rotator and a waveplate placed between two polarizing beam splitters (PBS). The fiber connecting the ports of PBS1 at the NOLM side is twisted by $90^{\circ}$, so that the pulse contribution transmitted at the entrance of the NOLM will be fully reflected at the exit, and vice versa. Therefore, the pulse contribution with linear polarization at $\pm 45^{\circ}$ will return onto itself when passing the NOLM loop through the Faraday rotator. The phase shift $\Delta \varphi$ of the waveplate WP, which is itself oriented at $45^{\circ}$, then leads to a nonreciprocal phase bias of $\Delta \varphi_{0}=2 \Delta \varphi$ per round trip. The splitting ratio of the NOLM may be tuned by changing the angular orientation of PBS2 or by an additional half wave plate in front of PBS2. This configuration presents the advantage that the splitting ratio can be tuned away from 50:50 without compromising the round-trip transmission of the laser. Furthermore, it can be used to fine-tune the splitting ratio according to the performance of the fiber loop which may vary due to manufacturing tolerances of fiber components or variable splice quality.

\section{Experimental results}

In the following, we report on three exemplary modelocked fiber lasers that have all been built in the novel reflective configuration as displayed in Fig. 1c. The lasers are operated at center wavelengths of 1030, 1565, and $2050 \mathrm{~nm}$, using Ytterbium (Yb), Erbium (Er), and co-doped Thulium-Holmium $(\mathrm{Tm} / \mathrm{Ho})$ as gain medium, respectively.

For all of the lasers, we have monitored the circulating laser light using a reflective beam splitter in the free-space optical path. Figure 2a shows the optical spectrum of an Erbium fiber laser with a repetition rate of $250 \mathrm{MHz}$. The laser is operated in the vicinity of zero dispersion with a small positive net value. Therefore, the optical spectrum is partially influenced by solitonic pulse shaping and partially by wavelength-dependent transmission of the fiber gain and optical components. The net dispersion is tailored using fibers of dispersion with opposite sign.

The spectrum has been taken with a resolution of $0.02 \mathrm{~nm}(2.46 \mathrm{GHz})$; therefore, the absence of spectral modulation excludes double pulses in the oscillator with a 


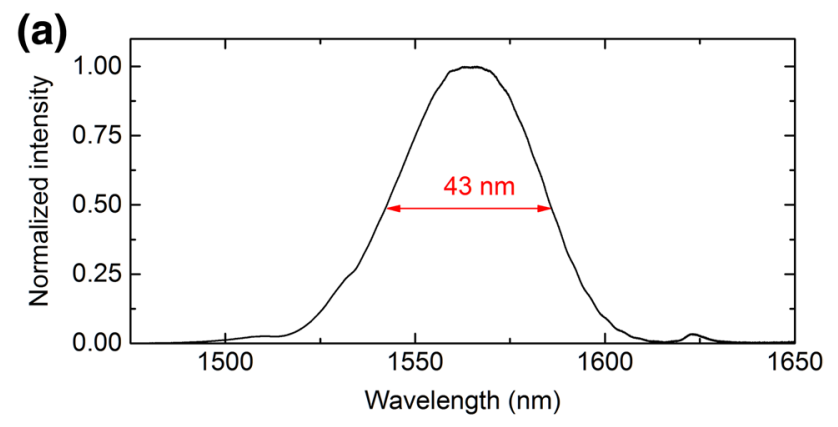

(b)

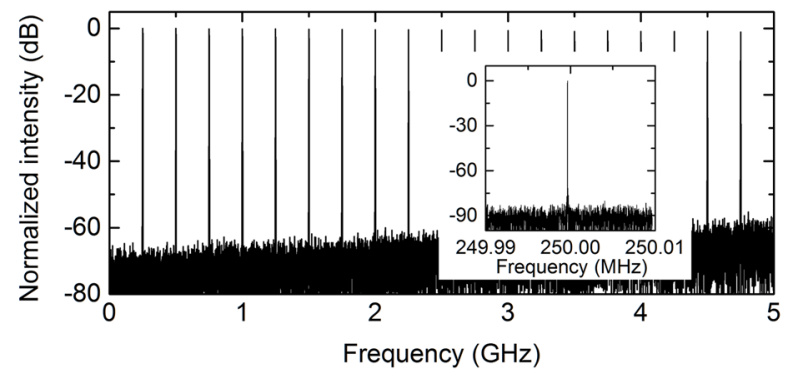

Fig. 2 Output characterization of a mode-locked Er fiber laser with a repetition rate of $250 \mathrm{MHz}$. a Optical spectrum (normalized). b RF spectrum of the laser power, measured with a resolution bandwidth of $1 \mathrm{MHz}$. The inset shows a close-up of the fundamental peak with a span of $20 \mathrm{kHz}$

separation smaller than $\sim 400$ ps. Furthermore, a fast photodiode has been used to sample the optical power in the time domain. Figure $2 \mathrm{~b}$ shows the corresponding RF spectrum from 0 to $5 \mathrm{GHz}$. Here, the absence of sinusoidal modulation excludes double pulses with a separation of more than 200 ps. Together, these measurements confirm single-pulse operation of the laser. Independently, the use of this laser as a frequency comb generator [15] attests single-pulse operation.

The observed relative intensity noise (RIN) shows a mostly constant floor between -130 and $-120 \mathrm{~dB} / \mathrm{Hz}$ for low frequencies, which we attribute to power and/or frequency fluctuations of the pump light. Above $10 \mathrm{kHz}$, these fluctuations are suppressed by the slow time constant of excitation relaxations in Erbium (Fig. 3).

As mentioned above, the novel reflective configuration has been successfully applied with other gain media and wavelengths. In the graph of Fig. 4, we show the optical spectrum of the Er-based laser together with exemplary spectra of an Yb-based laser at $1030 \mathrm{~nm}$ and Tm/Ho-based laser at $2050 \mathrm{~nm}$, respectively. The laser at $1030 \mathrm{~nm}$ is operated at a repetition rate of $100 \mathrm{MHz}$, and the laser at $2050 \mathrm{~nm}$ features a repetition rate of $10 \mathrm{MHz}$. While tuning of net dispersion at telecom wavelength or in the $2-\mu \mathrm{m}$ regime is easily achieved by combination of fibers with normal and anomalous dispersion, standard fibers in the vicinity of $1 \mu \mathrm{m}$ do not offer this possibility. Therefore, the

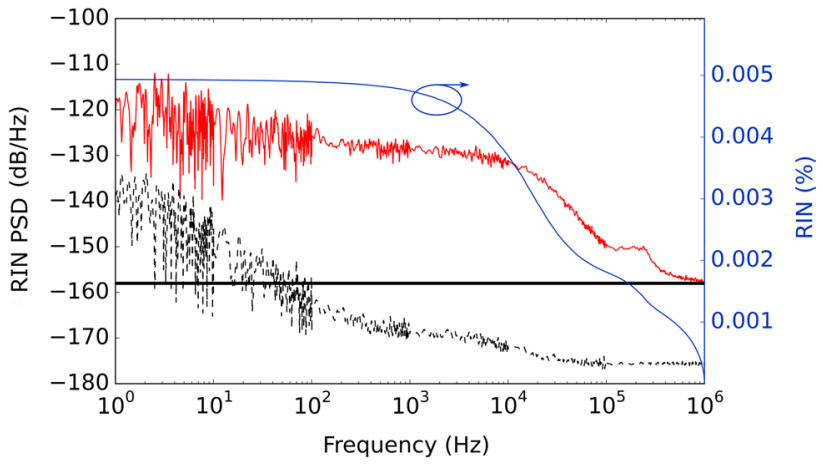

Fig. 3 Relative intensity noise (RIN) of the mode-locked Er fiber laser. Left axis power spectral density of the RIN (red, straight) and measurement noise floor (black, dashed), right axis integrated RIN (blue) as computed from the corresponding $x$-axis value to $1 \mathrm{MHz}$. The black horizontal line indicates the shot-noise limit corresponding to the average optical power impinging on the photodiode

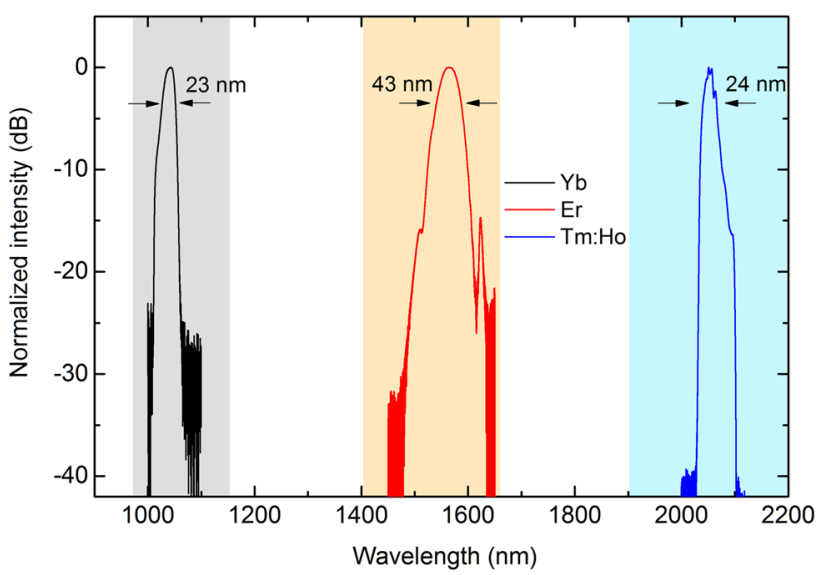

Fig. 4 Normalized optical spectra of three fiber lasers mode-locked with the novel reflective Sagnac loop, using $\mathrm{Yb}, \mathrm{Er}$, and $\mathrm{Tm} / \mathrm{Ho}$ as gain media. The shadowed area behind the spectra indicates the typical gain region of the corresponding gain medium. A close-up of all spectra in logarithmic scale can be found in the online material

free-space section of the $\mathrm{Yb}$ laser has been complemented by a grating pair and tuned to a dispersion regime similar to the one described for the Erbium laser. The individual spectra exhibit a 3-dB width of $23 \mathrm{~nm}(\mathrm{Yb}), 43 \mathrm{~nm}(\mathrm{Er})$ and $24 \mathrm{~nm}(\mathrm{Tm} / \mathrm{Ho})$ and support a Fourier-limited pulse width of $(84,72,240) \mathrm{fs}$, respectively. More details about the $\mathrm{Tm} /$ Ho system can be found in [16].

All of these oscillators are scaled to produce an output in the milliwatt regime [Yb: $10 \mathrm{~mW}$, Er: $3 \mathrm{~mW}, \mathrm{Tm} / \mathrm{Ho}$ : $4.6 \mathrm{~mW}$ with respective pump powers of approximately $215 \mathrm{~mW}(974 \mathrm{~nm}), 280 \mathrm{~mW}(974 \mathrm{~nm})$, and $1.7 \mathrm{~W}(790 \mathrm{~nm}$, double-clad pumped)]. Single-pulse operation is typically obtained in a pump range of $\pm 15 \%$ around a center value, and for pump power above the single-pulse regime, either cw-breakthrough or double-pulse operation is observed. 


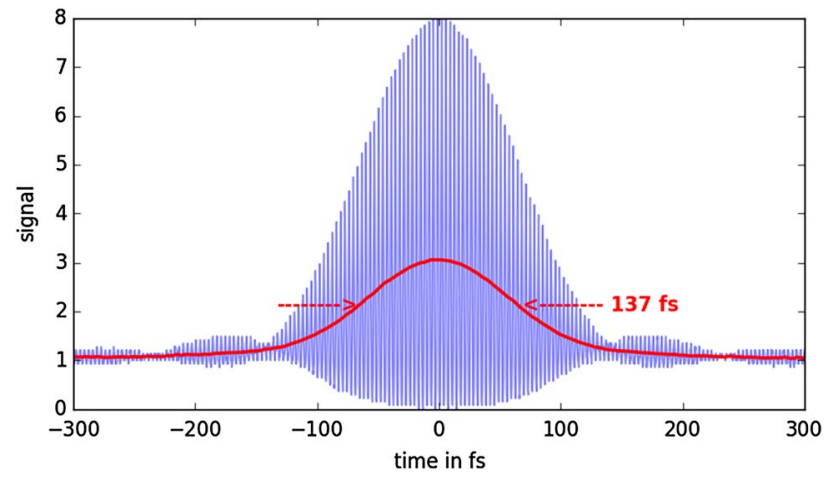

Fig. 5 Normalized autocorrelation trace of a femtosecond pulse from a $100 \mathrm{MHz}$ Yb laser with reflective Sagnac loop. Thin, blue interferometric autocorrelation trace; thick, red intensity autocorrelation trace. The output from the laser has been compressed by $16 \mathrm{~cm}$ of SF6 glass before analysis. Assuming a Gaussian pulse shape, the width of the intensity autocorrelation trace of $137 \mathrm{fs}$ corresponds to a pulse duration of $98 \mathrm{fs}$

For the $\mathrm{Yb}$ laser with repetition rate of $100 \mathrm{MHz}$, the peak intensity is high enough that an autocorrelation signal can be obtained without prior amplification (see Fig. 5). The signal is coupled out by a free-space component and compressed in $16 \mathrm{~cm}$ of SF6 glass. The measured autocorrelation width of $137 \mathrm{fs}$ points to a pulse width of $98 \mathrm{fs}$ (FWHM) assuming a Gaussian pulse shape. The deviation from the Fourier limit is explained by a slight third-order dispersion which also causes the small side peaks in the autocorrelation.

\section{Conclusion}

All lasers have proven to work reliably over months, serving as stable sources for the generation of terahertz radiation, as seed oscillators for material processing, for dielectric laser acceleration of electrons [17] and for the generation of highly pure microwave radiation [18]. At Menlo, we are particularly proud that these oscillators have also become the back bone of our new frequency comb systems. The reflective loop configuration with polarizationmaintaining fiber provides operation with very low intrinsic phase noise [15], surpassing the performance of fiber lasers mode-locked through NPR or semiconductor saturable absorbers [19]. Together with a novel electro-optic actuator for the carrier-envelope-offset frequency, the new frequency comb systems rival the performance of Ti: Sa-based systems [20], while the robustness of the PM fiber technology permits operation in rough environments and has even facilitated the first optical frequency comb in space [21].

We know it has always been a dream of Ted Hänsch that frequency comb technology becomes available in a simple and compact device. At Menlo, we have been inspired by his visions and his passion, and we hope that our efforts are helping to make his dream come true. Therefore, we dedicate this paper to Ted Hänsch in honor of his 75th birthday.

Acknowledgements We gratefully acknowledge funding from the BMWi (Project ZIM-TERA), the BMBF (Projects ExtruTera, DiaTumor, and Mirandus), the DLR (Projects FOKAL and IRASSI), the EU FP7 initial training network FACT, and the DARPA PULSE program (Project P $\mu$ reComb).

Open Access This article is distributed under the terms of the Creative Commons Attribution 4.0 International License (http://creativecommons.org/licenses/by/4.0/), which permits unrestricted use, distribution, and reproduction in any medium, provided you give appropriate credit to the original author(s) and the source, provide a link to the Creative Commons license, and indicate if changes were made.

\section{References}

1. T. Udem, J. Reichert, R. Holzwarth, T.W. Hänsch, Absolute optical frequency measurement of the cesium D1 Line with a mode locked laser. Phys. Rev. Lett. 82, 3568-3571 (1999)

2. E.P. Ippen, H.A. Haus, L.Y. Liu, Additive pulse mode locking. J. Opt. Soc. Am. B 6, 1736-1745 (1989)

3. K. Kieu, F.W. Wise, All-fiber normal-dispersion femtosecond laser. Opt. Express 16, 11453-11458 (2008)

4. N. Kuse, J. Jiang, C.-C. Lee, T.R. Schibli, M.E. Fermann, All polarization-maintaining Er fiber-based optical frequency combs with nonlinear amplifying loop mirror. Opt. Express 24, 3095$3102(2016)$

5. N. Raabe, M. Mero, Y. Song, W. Hänsel, R. Holzwarth, A. Sell, A. Zach, and G. Steinmeyer, Detecting determinism in laser noise: a novel diagnostic approach for ultrafast lasers. In Conference on Lasers and Electro-Optics (p. SM3I.5). (OSA, Washington, DC, 2016)

6. M. Hofer, M.E. Fermann, F. Haberl, M.H. Ober, A.J. Schmidt, Mode locking with cross-phase and self-phase modulation. Opt. Lett. 16, 502-504 (1991)

7. K. Tamura, H.A. Haus, E.P. Ippen, Self-starting additive pulse mode-locked erbium fibre ring laser. Electron. Lett. 28, 22262228 (1992)

8. I.N. Duling III, All-fiber ring soliton laser mode locked with a nonlinear mirror. Opt. Lett. 16, 539-541 (1991)

9. M.E. Fermann, F. Haberl, M. Hofer, H. Hochreiter, Nonlinear amplifying loop mirror. Opt. Lett. 15, 752-754 (1990)

10. H.A. Haus, E.P. Ippen, K. Tamura, Additive-pulse modelocking in fiber lasers. IEEE J. Quantum Electron. 30, 200-208 (1994)

11. J.W. Nicholson, M. Andrejco, A polarization maintaining, dispersion managed, femtosecond figure-eight fiber laser. Opt. Express 14, 8160-8166 (2006)

12. E. Baumann, F.R. Giorgetta, J.W. Nicholson, W.C. Swann, I. Coddington, N.R. Newbury, High-performance, vibration-immune, fiber-laser frequency comb. Opt. Lett. 34, 638-640 (2009)

13. W. Hänsel, R. Holzwarth, R. Doubek, M. Mei, Laser with nonlinear optical loop mirror. Eur. Pat. Appl. EP 2637265, A1 (2013)

14. T. Jiang, Y. Cui, P. Lu, C. Li, A. Wang, Z. Zhang, All PM fiber laser mode locked with a compact phase biased amplifier loop mirror. IEEE Photonics Technol. Lett. 28, 1786-1789 (2016)

15. W. Hänsel, M. Giunta, K. Beha, M. Lezius, M. Fischer, R. Holzwarth, Ultra-low phase noise all-PM Er: fiber optical frequency comb. In Advanced Solid State Lasers (p. ATh4A.2). (OSA, Washington, DC, 2015) 
16. H. Hoogland, S. Wittek, W. Hänsel, S. Stark, R. Holzwarth, Fiber chirped pulse amplifier at $2.08 \mu \mathrm{m}$ emitting 383 -fs pulses at $10 \mathrm{~nJ}$ and $7 \mathrm{MHz}$. Opt. Lett. 39, 6735-6738 (2014)

17. H. Hoogland, J. McNeur, M. Kozák, P. Hommelhoff, R. Holzwarth, Compact ultrashort pulsed $2.05 \mu \mathrm{m}$ all-PM fiber laser for dielectric laser acceleration of non-relativistic electrons. In Conference on Lasers and Electro-Optics, OSA Technical Digest ( $p$. SF1I.7). (OSA, Washington, DC, 2016)

18. X. Xie, R. Bouchand, D. Nicolodi, M. Giunta, W. Hänsel, M. Lezius, A. Joshi, S. Datta, C. Alexandre, M. Lours, P.-A. Tremblin, G. Santarelli, R. Holzwarth, Y. Le Coq, Photonic microwave signals with zeptosecond-level absolute timing noise. Nat Photonics. doi:10.1038/nphoton.2016.215

19. N. Kuse, C.-C. Lee, J. Jiang, C. Mohr, T.R. Schibli, M.E. Fermann, Ultra-low noise all polarization-maintaining $\mathrm{Er}$ fiber-based optical frequency combs facilitated with a graphene modulator. Opt. Express 23, 24342-24350 (2015)

20. M. Giunta, W. Hänsel, K. Beha, M. Fischer, M. Lezius, R Holzwarth, Ultra low noise Er: fiber frequency comb comparison. In Conference on Lasers and Electro-Optics (p. STh4H.1). (OSA, Washington, DC, 2016)

21. M. Lezius, T. Wilken, C. Deutsch, M. Giunta, O. Mandel, A. Thaller, V. Schkolnik, M. Schiemangk, A. Dinkelaker, A. Kohfeldt, A. Wicht, M. Krutzik, A. Peters, O. Hellmig, H. Duncker, K. Sengstock, P. Windpassinger, K. Lampmann, T. Hülsing, T. W. Hänsch, and R. Holzwarth, Space-borne Frequency Comb Metrology. Optica, 3, 1381-1387 (2016) 